\title{
Composition and the Logic of Location: An Argument for Regionalism
}

\author{
CODY GILMORE \\ UC Davis \\ gilmore@ucdavis.edu \\ MATT LEONARD \\ University of Southern California \\ matt523@usc.edu
}

\begin{abstract}
Ned Markosian (2014) has recently defended a new theory of composition, which he calls regionalism: some material objects $x x$ compose something if and only if there is a material object located at the fusion of the locations of $x x$. Markosian argues that regionalism follows from what he calls the subregion theory of parthood (STP). Korman and Carmichael (2016) agree. We provide countermodels to show that regionalism does not follow from (STP), even together with fourteen potentially implicit background principles. We then show that regionalism does follow from five of those background principles together with (STP) and two additional principles connecting parthood and location, which we call (Overlap) and (Strong Delegation). While the additional principles are not uncontroversial, our conjecture is that many will find them attractive. We conclude by mentioning that (Strong Delegation) fills a previously unnoticed gap in the formal theory of location presented in Parsons (2007).
\end{abstract}

\section{Introduction}

Ned Markosian (2014) defends a new answer to the special composition question: Under what circumstances do several material objects compose something? Markosian's new answer is regionalism: 'Necessarily, for any $x \mathrm{~s}$, there is a $y$ composed of those $x \mathrm{~s}$ iff there is a region, $r$, and an object, $z$, such that $r$ is the fusion of the regions occupied by the $x \mathrm{~s}$ and $z$ occupies $r$ ' (2014: 82). ${ }^{1}$ According to regionalism, some material objects compose something if and only if a material object is located at the fusion of their locations. For example, two hydrogen atoms and an oxygen atom compose something if and only if some material object (a water molecule, perhaps) is located at the fusion of those atoms' locations.

\footnotetext{
${ }^{1}$ Markosian adds that regionalism should be understood as entailing the ground-theoretic thesis that if some material objects compose something, then they compose something in virtue of the fact that some material object is located at the fusion of their locations (2014: 83). However, as he notes, he argues only for the simpler, non-groundtheoretic thesis. We too will set ground theory aside. Our results here are necessary preliminaries to any argument for the ground-theoretic thesis.
} 
Regionalism is consistent with a number of more familiar answers to the special composition question. This is because for any particular things $x x$ regionalism doesn't tell us whether a material object is located at the fusion of the locations of $x x{ }^{2}$ Markosian's own view is that composition 'occurs sometimes but not always'. To use his example: the particles that make up your brain do compose something (your brain), but your brain and Jeremy Bentham's body do not compose anything. Regionalism is consistent with this because it is consistent with the thesis that though there is a material object (your brain) located at the fusion of the locations of your brain's particles, there is no material object (not even a scattered object) located at the fusion of the locations of your brain and Bentham's body. Regionalism is also consistent with universalism about composition, the view that for any material objects $x x$, some $y$ is composed of $x x$. In fact, regionalism is consistent even with nihilism, the view that no complex objects exist.

One might complain (with Korman and Carmichael 2016) that this makes regionalism uninformative. Again, regionalism doesn't tell us which regions have complex material objects located at them and thus doesn't tell us whether any particular objects compose a further object. However, we think that regionalism is nevertheless an important thesis and that it is worthwhile to articulate the best argument for it. Unlike the more familiar answers to the special composition question, regionalism tells us something about complex material objects, their parts, and their locations. Since the familiar extant theories of composition say nothing about location, none of them guarantees both regionalism's necessity direction (that if some material objects $x x$ compose something, then a material object is located at the fusion of the locations of $x x$ ) and its sufficiency direction (that if a material object is located at the fusion of the locations of material objects $x x$ then $x x$ compose something). ${ }^{3}$

But both directions are tempting. Consider necessity. If material objects $x x$ compose something, $y$, it is plausible that some material object is located at the fusion of the locations of $x x$, namely, $y$ ! Where else would $y$ be? Two miles to the left? Platonic Heaven? Now consider the sufficiency direction. If a material object $y$ is located at the fusion of the locations of material objects $x x$, many will insist that $x x$ must compose something, namely, $y$. How could $y$ be so located without being composed of $x x$ ?

Even if regionalism does not settle the dispute between the nihilist, the moderate, and the universalist, it is an attractive thesis in its own right, and it imposes mereological and locational constraints on the more familiar theses. So we think that anyone who is interested in composition ought to be interested in what an argument for regionalism would need to look like.

Markosian argues that regionalism follows from what he calls the subregion theory of parthood (STP): for any material objects $x$ and $y$, ' $x$ is a part of $y$ iff the region occupied by $x$ is

\footnotetext{
${ }^{2}$ Regarding terminology, our notation for plural variables is ' $x x$ ', etc., whereas Markosian's is ' $x \mathrm{~s}$ ', and we use 'material object' as a synonym for Markosian's 'physical object'.

${ }^{3}$ Universalism does entail the sufficiency direction, by entailing that composition always occurs. Nihilism, together with the assumption that every material object has a location, entails the necessity direction, by entailing that if material objects $x x$ compose $y$, then each of $x x$ is identical to $y$.
} 
a subregion of the region occupied by $y^{\prime}(2014: 73) .{ }^{4}$ As we will see, regionalism is ambiguous between two natural readings. We will show that on neither reading does regionalism follow from (STP); on one reading, neither direction of regionalism follows from (STP). After we provide the countermodels that establish this, we set out a new argument that yields both versions of regionalism. The new argument relies on (STP) but also on several additional principles, some of which have been discussed in the literature. These additional principles are not uncontroversial. However, we conjecture that many will find them suitable for use in an argument for regionalism.

\section{Preliminaries}

We begin by setting out some assumptions and definitions. We use the language of plural logic, supplemented with a primitive two-place predicate for exact location, ' $x @ y$ ', and primitive oneplace predicates for regionhood, ' $R x$ ', and material objecthood, ' $M x$ '. We give the standard gloss of exact location: a material object $x$ is exactly located at a region $y$ just in case $x$ has the same size and shape as $y$ and bears the same distance relations to things as does $y$. For example, if o is a material object that is cubical, 1 cubic meter in volume, and 2 meters from object $\mathrm{o}^{*}$, then o is exactly located at a region $r$ only if $r$ is also cubical, 1 cubic meter in volume, and 2 meters from $\mathrm{O}^{*}{ }^{5}$

We also invoke a primitive two-place predicate, ' $x \leq y$ ', for parthood (being a proper-orimproper part of), together with predicates for proper parthood, overlapping, and disjointness, all defined in the usual ways: for $x$ to be a proper part of $y$ (' $x<y$ ') is for $x$ to be a part of but not identical to $y$; for $x$ to overlap $y$ (' $x \circ y$ ') is for something to be a part of both $x$ and $y$; and for $x$ and $y$ to be disjoint (' $x<y$ ') is for $x$ and $y$ to not overlap. We take our parthood predicate to apply univocally both to material objects and to regions, but everything we say could be restated in terms of a parthood predicate for material objects and a subregionhood predicate for regions. We formalize (STP) as follows: ${ }^{6}$

\footnotetext{
${ }^{4}$ Markosian (2014: 82-83) attempts a two-part proof of regionalism from (STP). In note 13 we identify the misstep.

${ }^{5}$ While talk of distance relations between points is straightforward, talk of distance relations between non-pointsized things is less so. But we hope that our gloss has some content and gives the reader a sense of the intended meaning of our primitive. (Others who treat exact location as a primitive and gloss it roughly as we do include Casati and Varzi (1999), Gibson and Pooley (2006), Gilmore (2006), Sattig (2006), Balashov (2010), Donnelly (2010), Saucedo (2011), Effingham (2015), and Lafrance (2015).) More informative characterizations can be given, but at a cost. For example, Parsons (2007) invokes a primitive notion of weak location, glossed as, ' $x$ is weakly located at $y$ iff $y$ is not completely free of $x$. He uses this primitive to define exact location: for $x$ to be exactly located at $y$ is for $x$ to be weakly located at all and only those entities that overlap (share a part with) $y$. This definition entails that if $x$ is exactly located at $r$ and also at $s$, then $r$ and $s$ overlap exactly the same things, which friends of multilocation (e.g., Sattig 2006) deny. However, our argument for regionalism rules out multilocation anyway, since it relies on Functionality (see $\$ 3$ ). So there is no harm in appealing to Parsons's characterization as a fallback option, for those who find our initial gloss of exact location insufficiently informative.
}

${ }^{6}$ A more literal translation of Markosian's principle into our formal language is (STP*):

$(M x \wedge M y) \rightarrow$ 


$$
(x @ r \wedge y @ s) \rightarrow(x \leq y \leftrightarrow r \leq s)
$$

(If $x$ is located at $r$ and $y$ located at $s$, then $x$ is a part of $y$ iff $r$ is a part of $s$.) ${ }^{7}$ (STP) is an 'alignment' principle, in Saucedo's (2011) terminology. It requires a kind of mirroring between, on one hand, the mereological relations between objects and, on the other, the mereological relations between the locations of those objects. ${ }^{8}$ We follow Markosian (and van Inwagen (1990)) and define ' $y$ is composed of $x x$ ' as:

$$
\begin{aligned}
& y C x x={ }_{d f} \forall w \forall z((w \prec x x \wedge z<x x \wedge w \neq z) \rightarrow w<z) \wedge \\
& \forall z(z \prec x x \rightarrow z \leq y) \wedge \forall z(z \leq y \rightarrow \exists x(x<x x \wedge z \circ x))
\end{aligned}
$$

(Composition)

( $y$ is composed of $x x=_{d f}(i)$ every two of $x x$ are disjoint, (ii) each of $x x$ is a part of $y$, and (iii) each part of $y$ overlaps at least one of $x x$.) As we mentioned, regionalism is ambiguous. Markosian frames the view in terms of fusions of regions. But there are different definitions of 'fusion' and Markosian does not say which he intends. So we will distinguish two versions of regionalism. The first version results from taking 'is a fusion of' to be synonymous with 'is composed of':

$$
\begin{aligned}
& (\forall x(x \prec x x \rightarrow M x) \wedge \forall w \forall z((w \prec x x \wedge z \prec x x \wedge w \neq z) \rightarrow w<z)) \rightarrow \\
& (\exists y(y C x x) \leftrightarrow \exists z \exists r \exists s s(r C s s \wedge M z \wedge z @ r \wedge \forall x(x \prec x x \rightarrow \exists s(s \prec s s \wedge x @ s)) \wedge \\
& \forall s(s \prec s s \rightarrow \exists x(x \prec x x \wedge x @ s))))
\end{aligned}
$$

(If each of $x x$ is a material object and every two of $x x$ are disjoint, then something is composed of $x x$ iff there is a $z$, an $r$, and some ss such that $(i) r$ is composed of $s s$, (ii) $z$ is a material object, (iii) $z$ is located at $r$, (iv) for each $x$ that is one of $x x$, there is an $s$ that is one of ss such that $x$ is located at $s$, and $(v)$ for each $s$ that is one of $s s$, there an $x$ that is one of $x x$ such that $x$

$(x \leq y \leftrightarrow \exists z \exists u(R z \wedge x @ z \wedge \forall w((R w \wedge x @ w) \rightarrow w=z) \wedge R u \wedge y @ u \wedge \forall w((R w \wedge y @ w) \rightarrow w=u) \wedge z \leq u))$

(if $x$ and $y$ are both material objects, then $x$ is a part of $y$ iff $x$ is located at exactly one region, $y$ is located at exactly one region, and the former region is a part of the latter region.) We prefer to work with (STP), which is more manageable. However, we evaluate the argument from (STP) to regionalism against background principles that guarantee, e.g., that all and only material objects have locations, that nothing has more than one location, and that only regions are locations. Nothing beneficial to Markosian's argument is lost when we shift from (STP*) to (STP) plus the background principles; our criticisms would carry over to a version of Markosian's argument that invokes (STP*).

${ }^{7}$ We use 'is located at' as a synonym of 'is exactly located at' throughout the paper. For readability, we omit initial, wide-scope universal quantifiers. We also omit initial necessity operators that take wide scope over the initial universal quantifiers. Nothing will turn on this. For each of the arguments we discuss, the non-modal version of the conclusion will be a logical consequence (in plural logic) of the non-modal versions of the premises iff the necessitation of the conclusion is a logical consequence (in any standard system of modal plural logic) of the necessitations of the premises.

${ }^{8}$ Varzi (2007), Uzquiano (2011), and Leonard (2016) discuss the logical relationships among many such principles. 
is located at s.) ${ }^{9}$ Less formally, $\left(\operatorname{Reg}_{C C}^{\leftrightarrow}\right)$ says that if $x x$ are disjoint material objects (' $D M x x$ '), then: $x x$ compose something ('CSxx') iff some material object is located at something that is composed of those objects' locations ('LCxx'). In short: $D M x x \rightarrow(C S x x \leftrightarrow L C x x)$.

The second version of regionalism, which we call $\left(\operatorname{Reg}_{C F}^{\leftrightarrow}\right)$, results from replacing the second occurrence of ' $C$ ' in the formal statement of $(\operatorname{Reg} \stackrel{\leftrightarrow}{C C})$ with a fusion predicate ' $F$ ' defined as follows:

$$
y F x x={ }_{d f} \forall z(z \prec x x \rightarrow z \leq y) \wedge \forall z(z \leq y \rightarrow \exists x(x \prec x x \wedge z \circ x))
$$

So defined, fusion is weaker than composition. From the claim that $y$ is composed of $x x$, it follows that $y$ is a fusion of $x x$. However, it follows from the former claim, but not from the latter claim, that no two of $x x$ overlap. (Henceforth, unless we explicitly say otherwise, by 'fusion' we will mean the notion defined by (Fusion).) Informally, (Reg $\leftrightarrow$ ) says that if $x x$ are disjoint material objects, then: $x x$ compose something iff some material object is located at something that is a fusion of those objects' locations ('LFxx'): $D M x x \rightarrow(C S x x \leftrightarrow L F x x)$.

Each version of regionalism is logically equivalent to a conjunction of two weaker principles, one offering necessary conditions for material composition, the other offering sufficient conditions. It will be convenient to have names for the four weaker principles. To minimize tedious formalism, we introduce them all at once, in abbreviated form:

Necessary condition for material composition

$(\operatorname{Reg} \overleftrightarrow{c C}) \quad$ equivalent to conjunction of
$(\operatorname{Reg} \overrightarrow{C C}): D M x x \rightarrow(C S x x \rightarrow L C x x) \quad$ and

$\Downarrow$

$\left(\operatorname{Reg}_{C F}^{\overleftrightarrow{C}}\right) \quad$ equivalent to conjunction of
$\left(\operatorname{Reg}_{C F}\right): D M x x \rightarrow(C S x x \rightarrow L F x x) \quad$ and

$(\operatorname{Reg} \overleftarrow{C F}): D M x x \rightarrow(C S x x \leftarrow L F x x)$

The weaker principles result from replacing the biconditional double-arrow in the formal statements of $\left(\operatorname{Reg} \overleftrightarrow{C C}\right.$ ) and $\left(\operatorname{Reg}_{C F}\right)$, respectively, with a single arrow (a material conditional or a converse material conditional, ' $\leftarrow$ ', as the relevant names indicate). The most important thing to

\footnotetext{
${ }^{9}$ Three points. First, the first clause in the antecedent is included because regionalism is only meant to supply conditions for material objects (not, say, regions) to compose something. Second, regarding the second clause of the antecedent, we follow Markosian, who writes that '[r]egionalism needs to be qualified so that it says "for any nonoverlapping $x \mathrm{~s}$..."' (2014: 82, note 26, emphasis original), in light of the disjointness clause in (Composition). Third, regarding ( $i i)$, we include it for the sake of explicitness, but given our assumption (and Markosian's) that only material objects have locations, it is redundant.
} 
see at this stage is that since fusion is weaker than composition, $(\operatorname{Reg} \overleftarrow{C C})$ is weaker than $\left(\operatorname{Reg}_{C F}^{\overleftarrow{C F}}\right)$ but $(\operatorname{Reg} \overrightarrow{C C})$ is stronger than $(\operatorname{Reg} \overrightarrow{C F})$.

Before moving on, we should note that at least two additional definitions of fusion commonly appear in the literature. ${ }^{10}$ These definitions are equivalent to (Fusion), given classical mereology, on which see Hovda (2009). While we will not assume classical mereology, we will make some assumptions about regions, which we take to be implicit in Markosian's discussion, that have a similar effect. Given these assumptions, the two additional versions of regionalism that result from the additional definitions of fusion are both equivalent to $(\operatorname{Reg} \stackrel{\leftrightarrow}{C F})$. So we will not give them separate treatment. ${ }^{11}$

\section{Countermodels}

Markosian (2014) and Korman and Carmichael (2016) claim that regionalism follows from (STP). In fact, neither $\left(\operatorname{Reg}_{C C}\right)$ nor $\left(\operatorname{Reg}_{C F}^{\leftrightarrow}\right)$ follows from (STP), even together with a generous collection of background principles that might be implicit in Markosian's discussion.

We divide the background principles into four groups. The principles in the first group, which constitute what Varzi (2016) calls Extensional Mereology, apply unrestrictedly to all entities, including regions and material objects.

\section{Extensional Mereology (EM)}

$x \leq x$

(Reflexivity)

Everything is a part of itself.

$(x \leq y \wedge y \leq z) \rightarrow x \leq z$

(Transitivity)

If $x$ is a part of $y$, and $y$ is a part of $z$, then $x$ is a part of $z$.

$(x \leq y \wedge y \leq x) \rightarrow x=y$

(Anti-symmetry)

If $x$ is a part of $y$, and $y$ is a part of $x$, then $x=y$.

\footnotetext{
${ }^{10}$ Our definition of 'fusion', which we take from from Lewis (1991), is what Hovda (2009) calls a 'Type-2 fusion', framed in plural logic. What Hovda (2009) calls 'Type-1 fusion' can be framed in plural logic as: $y$ is a T1-fusion of $x x$ iff: for all $z, z$ overlaps $y$ iff $z$ overlaps one of $x x$. Finally, the minimal upper bounds (MUB)-style definition, in plural logic, is: $y$ is a MUB-fusion of $x x$ iff each of $x x$ is a part of $y$ and for all $z$, if each of $x x$ is a part of $z$, then $y$ is a part of $z$. (For useful discussion, see Cotnoir 2016.) In the presence of assumptions (1) - (5) and (12) in the main text below, the versions of regionalism that result from interpreting ' $\mathrm{F}$ ' as $\mathrm{T} 1$-fusion or as MUB-fusion are equivalent to $(\operatorname{Reg} \overleftrightarrow{C F})$. We omit the proof.

${ }^{11}$ Fine (2010) and Kleinschmidt (forthcoming) argue, on different grounds, that 'fusion' is primitive and 'part' is defined. We lack the space to discuss how this shift would affect our project.
} 
If $x$ is not a part of $y$, then $x$ has some part $z$ that is disjoint from $y$.

Like classical mereology, EM entails that no plurality has more than one fusion. (Proof omitted.) But whereas classical mereology entails that every plurality has at least one fusion, EM leaves that question open. Thus, unlike classical mereology, EM avoids prejudging the answer to the special composition question.

The principles in the second group, which Markosian (2014: 86) seems to endorse, connect parthood with regionhood.

\section{Parts and Regions (PAR)}

$$
(x \prec x x \rightarrow R x) \rightarrow \exists y[y F x x \wedge \forall z(z F x x \rightarrow R z)]
$$

If each of $x x$ is a region, then: there is at least one fusion of $x x$ and any fusion of $x x$ is a region.

$$
(x \leq y \wedge R y) \rightarrow R x
$$

Any part of a region is a region.

PAR guarantees that every plurality of regions has at least one fusion. Together with EM, this entails that every such plurality has exactly one fusion. This leaves it open whether every plurality of material objects has a fusion. ${ }^{12}$

The principles in the third group concern regionhood, material objecthood, and their interaction with each other and with parthood.

\section{Material Objects and Regions (MOR)}

$$
[(x \prec x x \rightarrow M x) \wedge y F x x] \rightarrow M y
$$

If each of $x x$ is a material object and $y$ is a fusion of $x x$, then $y$ is a material object.

Nothing is both a region and a material object.

$$
(x \leq y \wedge M y) \rightarrow M x
$$

Any part of a material object is a material object.

These principles enforce a kind of dualism about material objects and regions.

\footnotetext{
${ }^{12}$ Although PAR plays no role in Markosian's argument or ours, we include it to make it explicit that even if it is assumed, Markosian's argument remains invalid. (R-Fusion) does however play a role in securing the equivalence of the additional versions of regionalism (mentioned in note 10$)$ to $(\operatorname{Reg} \overleftrightarrow{C F})$
} 
The principles in the fourth group, which we take to be endorsed by Markosian and in some cases implicit in his argument, concern location and its interaction with material objecthood and regionhood.

\section{Material Objects, Regions, and Location (MRL)}

$M x \rightarrow \exists y(x @ y)$

(Exactness)

Each material object has a location.

(11) $\exists y(x @ y) \rightarrow M x$

(Objecthood)

Only material objects have locations.

$\exists x(x @ y) \rightarrow R y$

(Regionhood)

All locations are regions.

$(x @ y \wedge x @ z) \rightarrow y=z$

(Functionality)

Nothing has more than one location.

$(x @ y \wedge z @ y) \rightarrow x=z$

(Anti-Colocation)

No two entities share a location.

MRL bans non-located material objects, multilocated entities, distinct co-located entities, and locations that are non-regions. It does not ban non-located entities in general, nor does it ban regions at which nothing is located.

Note that MRL says nothing about parthood. In particular, it says nothing about how the mereological properties of or relations between some objects must mirror the mereological properties or relations between the locations of those objects. Making these 'mirroring' requirements precise is the job of alignment principles such as (STP), which we treat as foreground, not background.

With (1) - (14) in place, we turn to our models. Each model is specified by a Hasse-style diagram. In each diagram, the solid nodes represent the material objects in the model and the hollow nodes represent the regions in the model. A straight upward-running line connecting a node $n$ to a higher node $m$ represents $n$ being a proper part of $m$. The downward-pointing arrows represent the higher node being located at the lower node. Keep in mind that a model need not correspond to a possible or even conceivable situation to make the desired point, which in each case is the purely logical point that a given sentence is a not a logical consequence of certain other sentences.

First we provide a model in which $(\mathrm{STP})$ and $(1)-(14)$ are true but $\left(\operatorname{Reg}_{C F}^{\overleftarrow{ }}\right)$ and $\left(\operatorname{Reg}_{C C}\right)$ are false. 


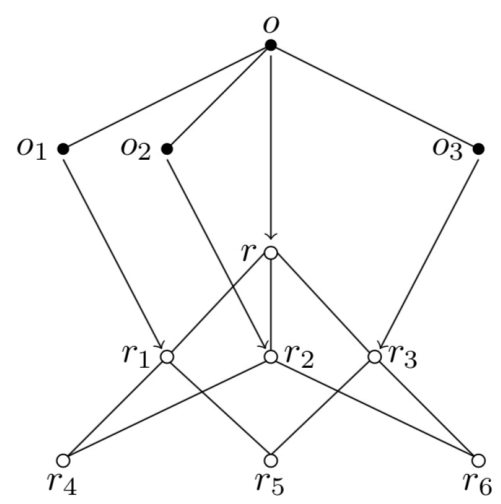

Figure 1

In this model, the complex material object $o$ is composed of the simple material objects $o_{1}, o_{2}$, and $o_{3} . o_{1}$ and $o_{2}$ do not compose anything. $o$ is located at $r, o_{1}$ is located at $r_{1}, o_{2}$ is located at $r_{2}$, and $o_{3}$ is located at $r_{3}$. As the reader can check, (STP) is satisfied in the model. For any case in which $x$ is located at $s_{1}$ and $y$ is located at $s_{2}, x$ is a part of $y$ iff $s_{1}$ is a part of $s_{2}$. (1) - (14) are also satisfied in the model, as the reader can also check. But while $r$ is a fusion of the locations of $o_{1}$ and $o_{2}$, and there is a material object located at $r$ in the model, $o_{1}$ and $o_{2}$ do not compose anything, so $(\operatorname{Reg} \overleftarrow{C F})$ is not satisfied. Though note that the model is not a countermodel to $(\operatorname{Reg} \overleftarrow{C C}): r$ is a fusion of $r_{1}$ and $r_{2}$, but $r_{1}$ and $r_{2}$ do not compose $r$, since they are not disjoint.

It is also important to see that $(\operatorname{Reg} \overrightarrow{C C})$ fails in the model. The material objects $o_{1}, o_{2}$, and $o_{3}$ do compose something, $o$, but no material object is located at something their locations compose. Their locations, which overlap each other, do not compose anything. Though note that $(\operatorname{Reg} \overrightarrow{C F}$ ) still holds: there is a material object located at $r$, which is the fusion of the locations of $o_{1}, o_{2}$, and $o_{3}$. So, whether we disambiguate regionalism via (Composition) or via (Fusion), our first model shows that regionalism does not follow from (STP) together with (1) - (14).

The distinctive feature of the model is that, although it satisfies (STP), it violates a similar-looking alignment principle:

$$
(x @ r \wedge y @ s) \rightarrow(x \circ y \leftrightarrow r \circ s)
$$

(If $x$ is located at $r$ and $y$ located at $s$, then $x$ overlaps $y$ iff $r$ overlaps $s$.) In the model, $o_{1}$ 's location overlaps the locations of $o_{2}$ and $o_{3}$, but $o_{1}$ overlaps neither $o_{2}$ nor $o_{3}$. It is tempting to assume that (Overlap) follows from (STP), but the above model shows that it does not. In fact, the two principles are logically independent, as noted by Uzquiano (2011).

We now provide a countermodel showing that neither $(\operatorname{Reg} \overrightarrow{C C})$ nor $(\operatorname{Reg} \overrightarrow{C F})$ follows from (STP) and (1) - (14). 


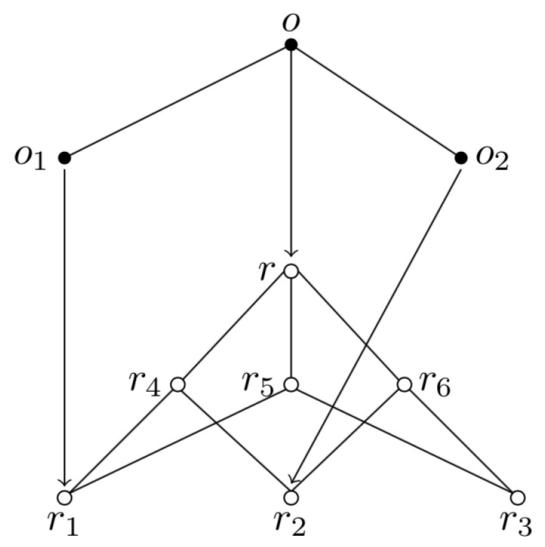

Figure 2

In this model, there are exactly three material objects. $o_{1}$ and $o_{2}$, are simple, and $o$ is complex: its parts are itself, $o_{1}$, and $o_{2} . o_{1}$ is located at $r_{1}, o_{2}$ is located at $r_{2}$, and $o$ is located at $r$, which is composed of $r_{1}, r_{2}$, and $r_{3}$. As the reader can check, (STP) and (1) - (14) are all satisfied in the model, as is (Overlap). Objects $o_{1}$ and $o_{2}$ compose something. Yet nothing is located at the region that is composed of - and fuses - their locations, $r_{4}$. So neither $(\operatorname{Reg} \overrightarrow{C C})$ nor $(\operatorname{Reg} \overrightarrow{C F})$ is satisfied. $^{13}$

The distinctive feature of this model is that although it satisfies (STP), (1) - (14), and (Overlap), it violates certain 'delegation' principles (so named by Gilmore (2009), (2018)). ${ }^{14}$ Delegation principles are alignment principles that say, roughly, that complex objects fill up their locations by delegation: if $r$ is the location of a complex object $o$, then no part of $r$ is filled by $o$ alone, without the help of some proper part ('delegate') of $o$. (To fill a region, let us say, is to have a location that has the given region as a part.) The delegation principle that will be our focus here is:

$$
\begin{aligned}
& (\exists y(y<x) \wedge x @ r) \rightarrow \\
& \forall y y\left(x F y y \rightarrow \forall r^{\prime}\left(r^{\prime} \leq r \rightarrow \exists s \exists y^{\prime}\left(y^{\prime} \prec y y \wedge y^{\prime} @ s \wedge r^{\prime} \circ s\right)\right)\right)
\end{aligned}
$$

\footnotetext{
13 This shows that Markosian's attempt to derive the necessity direction of regionalism from (STP) must go wrong somewhere but does not specify where. For those who are interested, here is the relevant passage from Markosian:

First we assume ... STP. Then we also assume of some randomly chosen $x$ s that there is an object composed of those $x \mathrm{~s} \ldots$. Call the object composed of the $x \mathrm{~s}$ A. It follows immediately from STP plus the fact that $\mathrm{A}$ is composed of the $x \mathrm{~s}$ that [M1] every one of the xs occupies a subregion of the region occupied by $A$. But from the fact that the $x \mathrm{~s}$ compose A we also know that [M2] there is no part of $A$ that fails to overlap at least one of the xs. So we know that [M3] the region occupied by $A$ is the fusion of the regions occupied by the $x$.' (2014: 83, italics added).
}

As our model shows, M3 does not follow from M1 and M2.

\footnotetext{
${ }^{14}$ Kleinschmidt (forthcoming) motivates her claim that 'fusion' is primitive in part by its ability to rule out similar scenarios, informally described. But the purely mereological system that results does not block our second model, so it seems that some delegation principle will still be needed.
} 
(If $x$ is complex and is located at $r$, then for any $y y$ of which $x$ is a fusion, each part $r^{\prime}$ of $r$ overlaps a location of one of $y y$.) Two comments. First, the idea is not that all objects must fill their locations by delegation. That would rule out simple material objects that have complex, extended locations. (For defenses of such objects, see Markosian (1998), Parsons (2000), and Simons (2004).) Second, our second model does violate (Strong Delegation). The object $o$ is complex and is a fusion of $o_{1}$ and $o_{2}$, but there is a part, $r_{3}$, of $o$ 's location that does not overlap a location of $o_{1}$ or of $o_{2}$.

One final remark about our models. We have stated (1) - (14) explicitly, and noted that our models satisfy all these principles, to dispel worries about the relevance of those models. The fact that the models satisfy (1) - (14) shows that those models target only the most relevant aspects of the argument. They do not trade on exotic possibilities about the behavior of parthood, as it applies to material objects or as it applies to regions. They do not appeal to distinct fusions of the same plurality, or failures of unrestricted fusion for regions, or multilocation, or distinct co-located entities, or non-located material objects. The only 'funny business' in the models is their violation of certain alignment principles. So, even against the background of a wellbehaved parthood relation and a separately well-behaved location relation, (STP) is not strong enough to deliver regionalism. Further alignment principles, ensuring that parthood and location interact as expected, are required. ${ }^{15}$

\section{The new argument}

Recall that there are two versions of regionalism on the table: $(\operatorname{Reg} \overleftrightarrow{C C})$ and $(\operatorname{Reg} \overleftrightarrow{C F})$. To prove that both versions follow from our premises, we will prove the stronger sufficient condition, $\left(\operatorname{Reg}_{C F}\right)$, and the stronger necessary condition, $\left(\operatorname{Reg}_{C C}\right)$. The countermodels discussed earlier satisfied (1) - (14). However, our argument will only rely on five such principles. We will refrain from invoking some of the more controversial principles, such as (Anti-Symmetry), (Strong Supplementation), (Exclusivity), (Objecthood), or (Anti-Colocation). Nor will we invoke (Transitivity), (R-Fusion), (Regional Parts), or (Regionhood), which are widely taken to be innocuous.

\subsection{Premises}

We will show that $(\operatorname{Reg} \overleftrightarrow{C C})$ and $\left(\operatorname{Reg}_{C F}^{\leftrightarrow}\right)$ are logical consequences of (STP), (Reflexivity), (MFusion), (Material Parts), (Exactness), and (Functionality), together with (Overlap) and (Strong Delegation), and an instance of the plural comprehension schema of plural logic. As attractive as

\footnotetext{
${ }^{15}$ We have allowed that (1) - (14) might be implicit premises in Markosian's argument. Why haven't we allowed that (Overlap) and (Strong Delegation) might be implicit premises too? First, even if (Overlap) and (Strong Delegation) are implicit premises, our models still show that regionalism does not follow from the set $\{(\mathrm{STP}),(1)-$ (14)\}, which was not antecedently obvious. Second, (Overlap) and (Strong Delegation) are more similar to (STP) than to any of (1) - (14). Like (STP), and unlike (1) - (14), they are alignment principles that govern the interaction of parthood and location. So, given that Markosian takes pains to make (STP) explicit, it seems that if (Overlap) and (Strong Delegation) were meant play a role in the argument, they would have been made explicit as well.
} 
these premises may be, they are not uncontroversial, and some objections to them are worth highlighting.

First, as Markosian notes, some think that it is possible for material objects (e.g., bosons) to interpenetrate without overlapping, like a ghost and a wall. (See Sanford (1967) and Gilmore (2018) for further references and discussion.) This would generate counterexamples to (Overlap) and (STP). Second, as Uzquiano (2011: 205) notes, even if one rejects ghost/wall-style interpenetration, one might think that disjoint, topologically closed material objects can be in perfect contact and have locations that share boundary points as common parts. This is inconsistent with (Overlap). Third, Gilmore (2006), Parsons (2007), Kleinschmidt (2016), and (Leonard 2014; 2018) note that in the context of non-standard models of space, one might think that a material object could fail to have an (exact) location. This is inconsistent with (Exactness). ${ }^{16}$ Fourth, some (e.g., Sattig 2006) think that material objects are multilocated. (See Donnelly (2010) and Gilmore (2018) for discussion.) This is inconsistent with (Functionality). Fifth, some (e.g., McDaniel (2001), Koslicki (2008)) think that some material objects have, as parts, 'formal components,' such as tropes or universals, that are not material objects. This is inconsistent with (Material Parts). Sixth, according to Cartwright (1975) and Uzquiano (2006), material objects can only be located at topologically open regions. Consider an open spherical object $o$ located at open spherical region $r$. $o$ is a fusion of two open hemispherical objects, $o_{1}$ and $o_{2}$, located at $r_{1}$ and $r_{2}$. Centerpoint $p$ of $r$ does not overlap $r_{1}$ or $r_{2}$ but is a part of $r$. This is inconsistent with (Strong Delegation).

So the argument will not carry weight with everyone. We suspect, however, that many will find the premises plausible. In any event, our goal is to chart connections, not to proselytize. Having shown that Markosian's argument is invalid, we seek further premises - the more plausible, the better - that would suffice to fill the gaps. Friends of the premises acquire a new route to regionalism; skeptics come to see how hard it is to repair Markosian's argument. Everyone learns something.

\subsection{Proof of validity}

Our proof comes in two steps: in the first we prove $\left(\operatorname{Reg}_{C F}^{\leftarrow}\right)$; in the second we prove $(\operatorname{Reg} \overrightarrow{C C})$

Step one. Recall that we abbreviated $\left(\operatorname{Reg}_{C F}^{\leftarrow}\right)$ as: $D M x x \rightarrow(C S x x \leftarrow L F x x)$. So, to prove this principle, we will consider some arbitrary things that satisfy ' $D M$ ' and ' $L F$ ', and we will show that they must satisfy ' $C S$ ' as well. Let $x x$ be arbitrary material objects each two of which are disjoint, and which are such that there is a $z$, an $r$, and some $s s$, such that (a) $r$ is a fusion of $s s$, (b) $z$ is a material object, (c) $z$ is located at $r$, (d) for each $x$ that is one of $x x$, there is an $s$ that is one of ss such that $x$ is located at $s$, and (e) for each $s$ that is one of $s s$, there is an $x$ that is one of $x x$ such that $x$ is located at $s$. We need to show that something $y$ is composed of $x x$. We will do this by showing that $z$ itself is composed of $x x$. Given the definition of composition,

\footnotetext{
${ }^{16}$ These authors actually discuss a related principle: if $x$ is weakly located somewhere, then $x$ is exactly located somewhere. But their cases also threaten our principle.
} 
we need to check that three conditions hold: ( $i$ ) each two of $x x$ are disjoint; (ii) each of $x x$ is a part of $z$; and (iii) every part of $z$ overlaps at least one of $x x$.

Condition ( $i$ ). This is true by hypothesis.

Condition (ii). Consider an arbitrary $a$ that is one of $x x$. By (d), $a$ is located at something, call it $s^{*}$, that is one of $s s$. Then, since $r$ is a fusion of $s s$, it follows by the definition of fusion that $s^{*}$ is a part of $r$. But since $a$ is located at $s^{*}, z$ is located at $r$, and $s^{*}$ is a part of $r$, it follows by (STP) that $a$ is a part of $z$. But $a$ was arbitrary, so condition (ii) is true.

Condition (iii). Assume for reductio that there is a part of $z$, call it $x^{\prime}$, that doesn't overlap any of $x x$. By (Material Parts), we know that $x^{\prime}$ is a material object. So by (Exactness), we know that $x^{\prime}$ must have a location, say, $s^{\prime}$. By hypothesis we know that $z$ is located at $r$. So $z$ is located at $r$ and $x^{\prime}$ is located at $s^{\prime}$ and $x^{\prime}$ is a part of $z$, from which it follows by (STP) that $s^{\prime}$ is a part of $r$. We also know, by hypothesis, that $r$ is a fusion of $s s$. By the definition of fusion, then, any part of $r$ overlaps at least one of $s s$. Recall $s^{\prime}$ is a part of $r$. So $s^{\prime}$ overlaps one of $s s$. But if $s^{\prime}$ overlaps one of $s s$ (which by hypothesis is a location of one of $x x$ ), then by (Overlap) we know that $x^{\prime}$ overlaps at least one of $x x$, which contradicts our initial assumption and completes our reductio. Therefore, condition (iii) holds. Since conditions $(i)-(i i i)$ hold, $(\operatorname{Reg} \overleftarrow{C F})$ is true.

Step two. Now we turn to $(\operatorname{Reg} \overrightarrow{C C})$, which we abbreviated as: $D M x x \rightarrow(C S x x \rightarrow L C x x)$. Consider arbitrary material objects $x x$ each two of which are disjoint and an arbitrary $y$ composed of $x x$. We need to show that there is a $z$, an $r$, and some ss such that five conditions hold: ( $i$ ) $r$ is composed of $s s,(i i) z$ is a material object, (iii) $z$ is located at $r$, (iv) for each $x$ that is one of $x x$, there is an $s$ that is one of ss such that $x$ is located at $s$, and $(v)$ for each $s$ that is one of $s s$, there an $x$ that is one of $x x$ such that $x$ is located at $s$.

Since each of $x x$ is a material object, by (Exactness), each of $x x$ has a location. Since something is a location of one of $x x$, by (Plural Comprehension) ${ }^{17}$ we know that there are some things that are all and only the locations of one or another of $x x .{ }^{18}$ Call these things $s s^{*}$. So: each of $x x$ is located at one of $s s^{*}$, and each of $s s^{*}$ is such that one of $x x$ is located at it, which means that $x x$ and $s s^{*}$ jointly satisfy $(i v)$ and $(v)$.

We can turn now to conditions ( $i$ ), (ii), and (iii). We know that $y$ is composed of $x x$. Hence we know, by the definitions of composition and fusion, that $y$ is a fusion of $x x$. We also know that each of $x x$ is a material object. It follows, by (M-Fusion), that $y$ is a material object. So, by (Exactness) and (Functionality), we know that $y$ has exactly one location, call it $L_{y}$. We'll show that $L_{y}$ is composed of $s s^{*}$, which will secure conditions (i), (ii), and (iii) and complete our proof.

\footnotetext{
${ }^{17}$ (Plural Comprehension): $\exists x \varphi(x) \rightarrow \exists y y \forall z(z \prec y y \leftrightarrow \varphi(z))$; if something is $\varphi$, there are some things $y y$ such that for all $z, z$ is one of $y y$ iff $z$ is $\varphi$.

${ }^{18}$ We substitute ‘ $\exists x^{\prime}\left(x^{\prime} \prec x x \wedge x^{\prime} @ x\right)$ ' for ‘ $\varphi(x)$ ' in (Plural Comprehension).
} 
We know that $y$ is composed of $x x$, hence that (a) each of $x x$ is a part of $y$. We also know that (b) $y$ is located at $L_{y}$. Finally, we know that (c) for each of $s s^{*}$, one of $x x$ is located at it. Together with (STP), (a) - (c) entail that (d) each of $s s^{*}$ is a part of $L_{y}$. So, in order to show that $L_{y}$ is composed of $s s^{*}$, it will suffice to prove two more things, namely, that (e) each part of $L_{y}$ overlaps at least one of $s s^{*}$; and that (f) each two of $s s^{*}$ are disjoint. We will prove (e) by cases, of which there are two.

Case one: $y$ does not have any proper parts. In other words, every part of $y$ is identical to $y$. So, given that $x x$ compose $y-$ and hence each of $x x$ is a part of $y-$ it follows that each of $x x$ is identical to $y$. So each of the locations of $x x$ - i.e., each of $s s^{*}-$ is identical to a location of $y$. We already know that there is exactly one location of $y$, namely, $L_{y}$. So each of $s s^{*}$ is identical to $L_{y}$. Trivially, each part of $L_{y}$ overlaps $L_{y}$. (This is guaranteed by (Reflexivity) and the definition of 'overlap'.) So, each part of $L_{y}$ overlaps at least one of $s s^{*}$ (namely, $L_{y}$ ). This means that (e) holds.

Case two: $y$ has at least one proper part. Then, since $y$ has a location (namely, $L_{y}$ ), the antecedent of (Strong Delegation) is satisfied, and we can conclude that (g) for any things $z z$ of which $y$ is a fusion and for any $u$, if $u$ is a part of $L_{y}$ then $u$ overlaps a location of one of $z z$. Now let $s^{\prime}$ be an arbitrary part of $L_{y}$. Since $y$ is a fusion of $x x$, and $L_{y}$ is a location of $y$, and $s^{\prime}$ is a part of $L_{y}$, by applying (g) we get the result that $s^{\prime}$ overlaps a location of one of $x x$; that is, $s^{\prime}$ overlaps one of $s s^{*}$. Since $s^{\prime}$ was arbitrary, we can conclude that each part of $L_{y}$ overlaps at least one of $s s^{*}:$ (e) holds.

So, whether $y$ has any proper parts or not, each part of $L_{y}$ overlaps at least one of $s s^{*}$. Given our earlier result that each of $s s^{*}$ is a part of $L_{y}$, it follows that $L_{y}$ is a fusion of $s s^{*}$. Now, to show that $L_{y}$ is composed of $s s^{*}$, all that remains is to prove that (f) each two of $s s^{*}$ are disjoint.

Assume for reductio that (f) is false, i.e., that there are non-identical but overlapping entities among $s s^{*}$ : call them $s_{i}$ and $s_{j}$. Since $s_{i}$ and $s_{j}$ are among $s s^{*}$, we know that one of $x x$, call it $x_{i}$, is located at $s_{i}$, and that one of $x x$, call it $x_{j}$, is located at $s_{j}$. Since $s_{i}$ and $s_{j}$ are nonidentical, we know by (Functionality) that $x_{i}$ and $x_{j}$ are non-identical. Since $x_{i}$ and $x_{j}$ are located at $s_{i}$ and $s_{j}$, respectively, and $s_{i}$ and $s_{j}$ overlap, it follows by (Overlap) that $x_{i}$ and $x_{j}$ overlap as well. But this contradicts our assumption that each two of $x x$ are disjoint. So we can conclude that (f) is true: each two of $s s^{*}$ are disjoint. It follows that $L_{y}$ is composed of $s s^{*}$.

We started with some arbitrary material objects $x x$ and an arbitrary $y$ composed of $x x$, and we showed that there is there is a $z$ (specifically, $y$ ) an $r$ (specifically, $y$ 's location, $L_{y}$ ), and some $s s$ (specifically, $x x$ 's locations, $s s^{*}$ ) such that five conditions hold: $(i) r$ is composed of $s s$, (ii) $z$ is a material object, (iii) $z$ is located at $r$, (iv) for each of $x x$ is located at one of $s s$, and $(v)$ each of ss is a location of one of $x x$. Since $x x$ and $y$ were arbitrary, $(\operatorname{Reg} \overrightarrow{C C})$ follows. 


\section{Conclusion}

We first showed that neither version of regionalism follows from (STP), even together with (1) (14). We then showed that both versions of regionalism follow from a different collection of premises, which includes (Overlap) and (Strong Delegation).

We close by noting a further application of (Strong Delegation). The influential theory of location presented in Parsons (2007) ${ }^{19}$ is satisfied by our second model. This shows, we think, that Parsons's theory is too weak. Though the theory may be true, it is not the whole truth about the logic of location. Any comprehensive formal theory of location should block our second model. The theory of Casati and Varzi (1999: 122) blocks the model with an axiom equivalent to (Arbitrary Partition): if $x$ is located at $r$ and $r^{*}$ is a part of $r$, then there is a part of $x$ that is located at $r^{*}$. However, since (Arbitrary Partition), together with (Functionality), rules out simple objects with complex, extended locations, which Parsons (2000), (2007) and Markosian (1998) want to allow, they reject (Arbitrary Partition). Without it, they are left with nothing to rule out our second model. We offer (Strong Delegation), which blocks that model but is friendly to simple objects with complex, extended locations. ${ }^{20}{ }^{21}$

\section{References}

Balashov, Yuri 2010, Persistence and Spacetime (Oxford: Oxford University Press). Cartwright, Richard 1975 'Scattered Objects', in Keith Lehrer (ed.), Analysis and Metaphysics (Dordrecht: Reidel), pp. 153-171.

Casati, Roberto and Achille C. Varzi 1999, Parts and Places: The Structures of Spatial Representation, (Cambridge, MA: MIT Press).

Cotnoir, A.J. 2016, 'Does Universalism Entail Extensionalism?', Noûs 50: pp. 121-132.

Donnelly, Maureen 2010, 'Parthood and Multi-location', Oxford Studies in Metaphysics, vol. 5: pp. 203-243.

Effingham, Nikk 2015, 'The Location of Properties', Nô̂s 49: pp. 25-44.

Fine, Kit 2010, 'Towards a Theory of Part', Journal of Philosophy 107: pp. 559-589.

Gibson, Ian and Oliver Pooley 2006, 'Relativisitic Persistence', Philosophical Perspectives, 20, Metaphysics: pp. 157-198.

Gilmore, Cody 2006, 'Where in the Relativistic World Are We?', Philosophical Perspectives, 20, Metaphysics: pp. 199-236.

\footnotetext{
${ }^{19}$ Specifically, the system, call it $\boldsymbol{S}++$, that results from supplementing what Parsons calls $\boldsymbol{S} @$ with what he calls Expansivity: $[(x \leq y \wedge x @ r) \rightarrow \exists s(y @ s \wedge r \leq s))] . \mathbf{S} @$ takes parthood and exact location as primitives and contains six axioms: the Asymmetry and Transitivity of proper parthood; Weak supplementation $[x<y \rightarrow \exists z(z<$ $y \wedge z<x)]$; Maximal common part $[x \circ y \rightarrow \exists z \forall w(w \circ z \leftrightarrow(w \leq x \wedge w \leq y))]$; (Functionality).

${ }^{20}$ Neither Casati and Varzi nor Parsons seek to rule out disjoint objects with overlapping locations, so they will see no need for a principle that blocks our first model. They will be skeptical of the right-to-left directions of the biconditionals in (STP) and (Overlap).

${ }^{21}$ We would like to thank the referees and editors for comments that improved the paper significantly.
} 
------ 2009, 'Why Parthood Might Be a Four Place Relation, and How it Behaves if it Is' in L.

Honnefelder, E. Runggaldier, and B. Schick, (eds.), Unity and Time in

Metaphysics (Berlin: de Gruyter, 2009), pp. 83-133.

2018, 'Location and Mereology', in The Stanford Encyclopedia of Philosophy (Spring 2018

Edition), Edward N. Zalta (ed.), URL =

$<$ https://plato.stanford.edu/archives/spr2018/entries/location-mereology/>.

Hovda, Paul 2009, 'What is Classical Mereology?', Journal of Philosophical Logic 38: pp. 55-

82.

Kleinschmidt, Shieva 2016, 'Placement Permissivism and Logics of Location', Journal of

Philosophy 113: pp. 117-136.

------ forthcoming, 'Fusion First' Noûs. doi:10.1111/nous.12236

Korman, Dan and Chad Carmichael 2016, 'Composition' in Oxford Handbooks Online. DOI: 10.1093/oxfordhb/9780199935314.013.9

Koslicki, Kathryn 2008, The Structure of Objects, (Oxford: Oxford University Press).

Lafrance, Jean-David 2015, 'A Bundle of Universals Theory of Material Objects', Philosophical Quarterly, 65: pp. 202-219.

Leonard, Matt 2014, 'Locating Gunky Water and Wine', Ratio, 27(3): pp. 306-315.

2016, 'What is Mereological Harmony?', Synthese, 193(6): pp. 1949-1965.

2018, 'Enduring through Gunk', Erkenntnis, 83(4): pp. 753-771.

Lewis, David 1991, Parts of Classes, (Oxford: Blackwell).

Markosian, Ned 1998, 'Simples', Australasian Journal of Philosophy, 76: pp. 213-226.

------ 2014, 'A Spatial Approach to Mereology', in Shieva Kleinschmidt (ed.) Mereology and

Location (Oxford: Oxford University Press), pp. 69-90.

McDaniel, Kris 2001, 'Tropes and Ordinary Physical Objects', Philosophical Studies 104: pp. 269-290.

Parsons, Josh 2000, 'Must a Four-Dimensionalist Believe in Temporal Parts?', The Monist, 83: pp. 399-418.

2007, 'Theories of Location', Oxford Studies in Metaphysics, vol. 3: pp. 201-232.

Sanford, David 1967, 'Volume and Solidity', Australasian Journal of Philosophy 45: pp. 329340.

Saucedo, Raul 2011, 'Parthood and Location', in Dean Zimmerman and Karen Bennett (eds.), Oxford Studies in Metaphysics, vol. 6: pp. 223-284.

Sattig, Thomas 2006, The Language and Reality of Time, (Oxford: Oxford University Press).

Simons, Peter 2004, 'Extended Simples: A Third Way Between Atoms and Gunk', The Monist, 87(3): pp. 371-384.

Uzquiano, Gabriel 2006, 'Receptacles', Philosophical Perspectives, 20: pp. 427-451.

------ 2011, 'Mereological Harmony', in Dean Zimmerman and Karen Bennett (eds.), Oxford

Studies in Metaphysics, vol. 6: pp. 199-224.

van Inwagen, Peter 1990, Material Beings, (Ithaca: Cornell University Press).

Varzi, Achille 2007, 'Spatial Reasoning and Ontology: Parts, Wholes, and Locations', in Marco Aiello Pratt-Hartmann, Ian E and Johan F.A.K. van Benthem, (eds.), Handbook of Spatial Logics (Berlin: Springer-Verlag), pp. 945-1038.

------ 2016, 'Mereology', in The Stanford Encyclopedia of Philosophy (Winter 2016 Edition), Edward N. Zalta (ed.), URL =

$<$ https://plato.stanford.edu/archives/win2016/entries/mereology/>. 